\title{
Administración pública y el presupuesto participativo en los gobiernos municipales de la Provincia de Santo Domingo-Ecuador
}

\section{Public administration and participatory budgeting in the municipal governments of Santo Domingo-Ecuador}

\author{
DOI: $10.46932 / \mathrm{sfjdv2n4-088}$
}

Received in: March 1st, 2021

Accepted in: May 30th, 2021

\author{
Félix Sixto Pilay Toala \\ Doctor en Proyectos, por la Universidad Internacional Iberoamericana de México \\ Magister en Administración Pública por la Universidad Tecnológica América \\ Institución: Pontificia Universidad Católica del Ecuador, Sede Santo Domingo, Ecuador \\ Dirección: Vía Chone Km 2, Santo Domingo, Ecuador, CP: 230203 \\ E-mail: ptfs@pucesd.edu.ec

\section{Mikel Ugando Peñate} \\ Doctor en Economía, por la Universidad de Santiago de Compostela, España \\ DEA en Programa Doctorado Convergencia Internacional en el Ámbito Financiero y Contable, por la \\ Universidad de Vigo, España \\ Institución: Pontificia Universidad Católica del Ecuador, Sede Santo Domingo, Ecuador \\ Dirección: Vía Chone Km 2, Santo Domingo, Ecuador, CP: 230203 \\ E-mail: mugandop@pucesd.edu.ec,ugando75@gmail.com \\ María Jahaira Álava Carvajal \\ Magister en Administración Pública por la Universidad Tecnológica América \\ Institución: Universidad San Gregorio de Portoviejo, Manabí, Ecuador. \\ Dirección: AV METROPOLITANA, Av. Olímpica, Portoviejo 130101, Ecuador, \\ E-mail: jahaira84alava@gmail.com
}

\section{RESUMEN}

La investigación tiene como objetivo determinar si los gobiernos municipales de la provincia de Santo Domingo de los Tsáchilas, Ecuador, como parte esencial de la administración pública elaboran el presupuesto anual de manera participativa, como lo determina la Constitución de la República, el Código Orgánico de Organización Territorial, Autonomía y Descentralización, Código Orgánico de planificación y Finanzas Públicas y el código orgánico de Participación Ciudadana. El desarrollo de la metodología se establece a través de un enfoque mixto, con una población de estudio en los cantones de Santo Domingo y La Concordia, se aplicaron 385 encuestas con un cuestionario estructurado y también semiestructurado a hombres y mujeres entre 18 y 69 años, se realizaron entrevistas a líderes de opinión tanto locales como nacionales; la técnica de observación, fue empleada en visitas a las zonas vulnerables, además se realizó una experimentación social. Dentro de los resultados, se evidencian las causas para el deficiente acceso a las necesidades prioritarias y las razones para que los recursos económicos que ingresan a la entidad municipal no son distribuidos de manera adecuada. Existe un marco legal propicio para que los gobiernos locales elaboren su presupuesto de forma participativa, de acuerdo a los datos obtenidos en la encuesta, existe carencia de un modelo de gestión simplificado.

Palabras Claves: Administración Pública, Gestión, Participación Social, Asignación de Recursos, Presupuesto del Estado 


\section{ABSTRACT}

The objective of the investigation is to determine whether the municipal governments of the province of Santo Domingo de los Tsáchilas, Ecuador, as an essential part of the public administration, prepare the annual budget in a participatory manner, as determined by the Constitution of the Republic, the Organic Code of Territorial Organization, Autonomy and Decentralization, Organic Code of Planning and Public Finance and the Organic Code of Citizen Participation. The development of the methodology is established through a mixed approach, with a study population in the cantons of Santo Domingo and La Concordia, 385 surveys were applied with a structured and semi-structured questionnaire to men and women between 18 and 69 years old. interviews were conducted with local and national opinion leaders; The observation technique was used in visits to vulnerable areas, and a social experimentation was also carried out. Among the results, the causes for the deficient access to the priority needs and the reasons for the economic resources that enter the municipal entity are not distributed adequately. There is a favorable legal framework for local governments to prepare their budget in a participatory way, according to the data obtained in the survey, there is a lack of a simplified management model.

Key words: Public administration, Management, Social participation, Resource allocation, State budget

\section{INTRODUCCIÓN}

El Presupuesto participativo es un instrumento apropiado para administración pública, la gestión administrativa y financiera en una entidad autónoma y descentralizada municipal. Representa a su vez, un instrumento eficaz en el proceso de fortalecimiento económico financiero que permite la cohesión de propósitos entre el gobierno municipal y la ciudadanía beneficiaria en especial de los sectores vulnerables. Además, es un instrumento de políticas públicas que se lo ha utilizado de manera eficaz en varios países del mundo.

En España, se estima que aproximadamente 140 ayuntamientos utilizan esta herramienta obteniendo con ello, impactos positivos; en Alemania, de igual manera y además existe una OMG denominada GTZ, que impulsa a nivel de ayuntamientos o municipios en varios países, la implementación de este sistema de administrar los recursos públicos.

En Ecuador, el presupuesto participativo como instrumento de la políticas públicas, administración y financiera, parte central de la democracia participativa, surge en tiempos de crecimiento de la corrupción y mal manejo de los fondos públicos, por las autoridades locales “Corrupción que han sufrido varios países y con los esfuerzos de las naciones Unidas por mejorar la gobernabilidad, la lucha contra la corrupción, el incremento de la trasparencia y la rendición de cuentas" (Buele y Vidueira, 2018, p.161).

Este instrumento de política pública, podría muy bien relacionarse con los objetivos 1 y 10 de los 17 objetivos de Desarrollo Sostenible de la Agenda 2030 de la Organización de las Naciones Unidas (2015) en donde el objetivo 1, busca poner fin a la pobreza en todas sus formas en todo el mundo; y el 10, que busca reducir la desigualdad en y entre los países. El modelo de gestión propuesto participativo se basa en el principio de redistribución del ingreso y en la descentralización autonómica en la capacidad de soluciones propias. 
Esta forma de gestión pública surge en Porto Alegre - Brasil, en esta parte del continente americano, por primera vez vio la luz, luego se fue expandiendo por todo el país y luego por varios países de la región como Argentina, México, Colombia, Chile, Perú, Bolivia y Venezuela entre otros, con el ingrediente de que en cada una de las naciones se lo diseña a su manera.

En América Latina, varios son los países en donde el presupuesto participativo ha sido implementado por los gobiernos locales, aunque de acuerdo a los diferentes autores citados en este trabajo, cada autoridad municipal lo ha implementado a su manera, pero sin embargo, el instrumento de gestión pública ha sido exitoso, como un mecanismo de fortalecimiento de la democracia "Los defensores de la democracia participativa destacan su papel en complementar y fortalecer la democracia representativa por dar voz a aquellos que de otra manera no serían escuchados" (Welp, 2016, p.105).

En Ecuador a pesar de que existe todo un marco jurídico para su implementación en las entidades municipales el presupuesto participativo, tales como la Constitución de la República del Ecuador (2008), el COOTAD (Código Orgánico de Organización Territorial Autonomía y Descentralización) (2011), Ley Orgánica de Participación Ciudadana (2010), Código Orgánico de Planificación y finanzas públicas (2010), sin embargo, los gobiernos autónomos descentralizados municipales no la cumplen en su totalidad.

Se puede evidenciar que los gobiernos locales no han utilizado adecuadamente la herramienta del presupuesto participativo o existe una deficiencia en su gestión, pese a lo imperativo de la ley, utilizan mecanismos no acorde con la participación de la ciudadanía, al parecer lo importante es no entrar en contradicción con la ley, antes que, resolver las necesidades prioritarias de los grupos humanos vulnerables, con lo cual se determina una deficiencia en su gestión, lo que podría ser considerado como falta de decisión política. Estas mismas deficiencias ocurren en los procesos de planificación estratégica por parte de los gobiernos según exponen Ugando, Jurado \& Cedeño (2021).

Es necesario destacar que, en el Ecuador, cuando nadie hablaba del presupuesto participativo, el Alcalde, Auki Tituaña del Cantón Cotacachi, “[...] implementó en el año 2000, el presupuesto participativo, lo interesante es que va más allá, de la simple incorporación de la ciudadanía a la acción pública, sino que además transfiere competencia en seguimiento y control [...]" (Arenilla Sáez, 2008, citado por Pilay 2015).

En Santo Domingo y La Concordia, ciudades escenarios de la investigación, la población vulnerable, no se siente representada en la toma de decisiones de los gobiernos locales, en lo concerniente a las obras prioritarias como agua potable, energía eléctrica, alcantarillado, canalización, vías de comunicación, centro de salud, entre otras, lo que conduce a los pobladores a que participen en grandes jornadas de movilizaciones para conseguir que los municipios desarrollen obras acorde a su competencia 
y en general son soluciones de menor cuantía. Estos critertios se encuentran expuestos y ejemplificados por Pilay \& Ugando (2020).

Se ha detectado que, en los Gobiernos Autónomos Descentralizados Municipales al persistir una inobservancia de la ley en la elaboración participativa del presupuesto anual, causa una deficiencia o carencia en la gestión administrativa y financiera, trayendo como consecuencia que los recursos que ingresan al Municipio ya sean propios, proveniente de los impuestos y tasas por mejoras o las asignaciones del Gobierno Central no sean distribuidos de manera, equitativa, participativa y solidaria.

A nivel internacional el presupuesto participativo ha cobrado una gran relevancia, es considerado por algunos investigadores como una forma adecuada de gestión de los recursos de la administración pública y de las políticas públicas, León, Hincapié y Villa (2016), Garrido, Montecinos, (2018) sostienen que el presupuesto participativo es una experiencia novedosa porque favorecen la expansión de la democracia directa.

El presupuesto anual elaborado de manera participativa ha sido y es una experiencia novedosa en América Latina, en Chile se han realizado varias investigaciones sobre este tema, uno de los más recientes es un artículo de investigación científica propuesto por Noriega, Aburto y Montecinos (2016) denominado "Presupuesto participativo en Chile y su contribución a la inclusión social", se mostraron importantes aportes de este ejercicio democrático tanto a la participación ciudadana como a la inclusión social. "El resultado evidenció que, en la práctica esta forma de administración pública no ha logrado incluir en su totalidad a toda la ciudadanía, Los hallazgos determinaron que son los jóvenes, los menos favorecidas en términos de participación".

Garrido y Montecinos, (2018) en un estudio realizado, para medir el comportamiento del presupuesto participativo en Chile y República Dominicana, reflexionan que "el presupuesto participativo es determinante para el fortalecimiento de la democracia participativa", al mismo tiempo sostienen que “el desarrollo del presupuesto participativo no tiene que ver con aspectos legales sino más bien con la voluntad política del alcalde y del rol activo de la organización social".

Nebot (2018) en una investigación intitulada "Presupuesto Participativo de Madrid: luces y sombra", analizó, los más de "25 años de funcionamiento del presupuesto participativo en américa y la nueva experiencia que se puso en marcha en el ayuntamiento de Madrid", revela que en algunos ayuntamientos en España, varios líderes de los gobiernos locales decidieron incorporar en sus programas de gobierno el presupuesto participativo como una forma para ganar eficiencia en la gestión administrativa y financiera.

El estudio del presupuesto participativo, fue y seguirá siendo un tema de interés para muchos investigadores, como se revelan evidencias en la investigación de Pilay \& Ugando (2020), cientistas 
sociales de diversos países de América Latina e incluso España en Europa han realizado investigaciones e importantes contribuciones sobre este tema, que tiene mucha significancia por ser una política pública que bien podría garantizar mejorar las condiciones de vida de sectores sociales olvidados por la acción de los gobiernos locales.

En América Latina se fue expandiendo la necesidad de impulsar esta nueva forma de administrar los recursos públicos a través del presupuesto participativo como una herramienta que transformaba al presupuesto de ser un instrumento puramente técnico, a una forma política, "para reforzar los vínculos entre las decisiones políticas y los ciudadanos, planteó la creación de un nuevo centro de decisión, que junto al poder ejecutivo y legislativo, fue democratizada la acción política e integrada a los ciudadanos comunes" (Díaz, 2011: 16).

Las entidades públicas municipales en América Latina, lejos de utilizar este instrumento como una actividad permanente para que la ciudadanía desarrolle experticias para elaborar el presupuesto participativo conjuntamente con sus autoridades, se observa que lo que han hecho es debilitar y detener su proceso de tal manera que las "innovaciones participativas terminan convirtiéndose en una manifestación temporal, muy frágil a los cambios electorales, y que no logran trascender en la sociedad civil ni en el entramado institucional permanente de decisión de las políticas públicas comunales o municipales" (Montecinos, 2012, p.1).

El presupuesto participativo que se implementó en la ciudad de Porto Alegre, Brasil, tuvo como objetivo lograr que las organizaciones sociales y la ciudadanía en su conjunto participen de manera activa y efectiva en la planificación y en la toma de decisiones juntamente con su liderazgo local, para garantizar mejores condiciones de vida. Esta forma novedosa de la administración pública y privada se la denominó en su tiempo como "La Administración Popular" (Harnecker, 1999, citado por Pilay 2015).

Ecuador al igual que los países en América Latina, presentan problemas de redistribución de la riqueza, las que se han transformado en graves desigualdades sociales y que son muy evidentes en los sectores poblacionales, considerados como zonas vulnerables y que se encuentran ubicados fuera de la influencia directa del Gobierno Central, por lo que, son atendidos por los GAD's, (gobiernos autónomos descentralizados), sean estos, provinciales, municipales y parroquiales.

En la investigación realizada en Quito-Ecuador, por Hidalgo (2017) manifestó que "los resultados dieron como evidencias que las asambleas ciudadanas en su ejecución y funcionamiento en cuanto a: la inclusión de actores periféricos, no existieron espacios de discusión, la forma de deliberación y los actores involucrados en el proceso que no se adaptan a las reglas establecidas" estos resultados serían según el autor, por ausencia de garantías para la distribución equitativa del presupuesto. 
Es urgente que los GAD's municipales del país y de América Latina se pongan a tono con los cambios sociales y políticos, hacer de las asambleas ciudadana un verdadero instrumento de participación de diversos actores que permita la articulación de políticas públicas “[...] El pasaje hacia prácticas más legítimas y efectivas en la resolución de problemas supone, cada vez más, un profundo replanteo de los vínculos tradicionales entre el Estado y la sociedad y la democratización de sus relaciones [...]” (Carmona y Martínez, 2014, p.298).

Los Gobiernos locales, de la provincia de Santo Domingo de los Tsáchilas, al igual que la mayoría de las entidades municipales del Ecuador, no existe ese vínculo necesario entre el Estado y la Sociedad, los gobiernos autónomos descentralizados municipales, no han gestionado el presupuesto participativo de manera adecuada a pesar de que en los meses previos a través de los medios de comunicación se informa que se elaborará el presupuesto de forma participativa, al parecer en los hechos no se realiza como se debe; por tanto, las obras de desarrollo prioritarios en los sectores vulnerables no se concretan y siguen postergadas.

\section{MATERIALES Y MÉTODOS}

Para responder a las preguntas de investigación y a los objetivos planteados en la investigación para determinar la administración pública y el presupuesto participativo en los gobiernos municipales de la Provincia de Santo Domingo, fue necesario el diseño experimental que implicó combinación investigativa tanto cualitativa como cuantitativa (Hernández y Mendoza, 2018: 625).

Además, se buscó en este diseño de tipo de investigación, obtener los paradigmas de los métodos teóricos, exploratorios y analíticos; de tal manera, que la información que se obtuvo fue objetiva; en concordancia a lo planteado por Hernández y Mendoza, (2018), fue un estudio de orden de experimentación de campo.

\subsection{ALCANCE DE LA INVESTIGACIÓN}

La metodología que se empleó en la investigación fue de acuerdo al planteamiento del problema, sus objetivos propuestos, de los métodos y técnicas a utilizarse en la recepción y recogida de los datos, mediante la aplicación de cuestionario de la encuesta, observación, en los cantones involucrados, Santo Domingo y la Concordia, realización de entrevistas a expertos y a líderes de opinión locales y nacionales y además de la revisión bibliográfica, en bibliotecas físicas del Ecuador y bases de datos digitales, fue conveniente utilizar el enfoque mixto de la investigación.

Se logró el objetivo trazado o la información deseada mediante la utilización de métodos exploratorios, descriptivos y un diseño experimental o explicativo. Mediante el enfoque cualitativo, se 
implementó las observaciones, estas fueron realizadas en el sitio, tanto en Santo Domingo como en la Concordia, en una interacción entre pobladores de los sectores vulnerables, para el cumplimiento del enfoque cuantitativo, se actuó sobre el mismo con encuestas que incluyeron preguntas cerradas y preguntas semicerradas, entrevistas semiestructuradas a expertos y observaciones a grupo focal.

\subsection{ANÁLISIS DE LA POBLACIÓN Y MUESTRA OBJETO DE ESTUDIO}

Los cantones de Santo Domingo y La Concordia, que conforman la provincia de Santo Domingo de los Tsáchilas, cuentan con una población estimada en el área urbana de 270,875 y de 29,003 habitantes respectivamente, según datos del INEC, del último censo de 2010, para efecto del trabajo investigativo para el caso de Santo Domingo por su condición de capital provincial no se consideró el total de sus habitantes, sino aquellos que se encuentra en las parroquias urbanas, en los sectores considerados como vulnerables.

Para el caso de estudio de la Concordia, se consideró el total de sus habitantes, por su número reducido y por su reciente creación, el cantón la Concordia, según datos obtenidos en la página del GAD’s, municipal, en el 2007, fue creado como cantón de la provincia de Esmeraldas y el 2013, mediante referéndum su población mayoritariamente decidió ser parte de la naciente Provincia de Santo Domingo de los Tsáchilas; por tanto, sus habitantes en su mayoría carecen de los servicios básicos, por lo que podría considerársela como vulnerable; por tanto, fue este grupo humano, quienes cumplieron con los criterios de exclusión, inclusión y delimitación.

La forma que se utilizó para la recolección de los datos de la unidad de estudio que estuvo conformada por individuos fue mediante las técnicas de documentación, de observación, de entrevista y finalmente de encuestas. Mediante la documentación se extrajeron datos secundarios, provenientes de libros, artículos de investigación, periódicos y revistas especializadas, los datos primarios se extrajeron de las encuestas en los sectores vulnerables, entrevistas a los alcaldes, vicealcaldes, directores departamentales y líderes de opinión, y de la observación, mediante asistencia a reuniones de cabildo que tuvieren lugar; de visita a los sectores vulnerables, de tal manera, que fue una medición lo más objetiva precisa y sin sesgo.

Para la obtención de la muestra, en las zonas vulnerables pertenecientes a Santo Domingo y La Concordia, se utilizó un muestreo probabilístico aleatorio simple infinito con un nivel de confianza del $95 \%$ y un factor probabilístico de $1.96^{2}$, el $5 \%$, de error de estimación al cuadrado, con una probabilidad de ocurrencia del $50 \%$ y con una probabilidad de no ocurrencia del 50\%, de lo que se obtuvo una muestra de 385 personas respectivamente, a partir de la fórmula de tamaño muestral infinita y finita que se detalla. 


\subsection{ANÁLISIS DEL INSTRUMENTO DE INVESTIGACIÓN}

Previo a la implementación del instrumento de la investigación, se procede a la validación por cinco expertos nacionales e internacionales, profesores de tres importantes universidades ecuatorianas, con formación académica $\mathrm{PhD}$, mediante el sistema denominado "juicio de expertos", quienes luego de examinar de manera individual la herramienta procedieron a emitir su criterio enfocado en el establecimiento de la relevancia, claridad y pertenencia, para lo cual, realizan observaciones y validan la implementación.

El instrumento diseñado por los autores para la recolección de datos, además fue sometida a la validación o análisis de confiabilidad mediante el sistema del valor Alfa de Cronbach, obteniendo como resultado un rango mayor a 0.8 y menor a 1.0, con lo cual, se obtiene una excelente confiabilidad del instrumento; una vez, validada y comprobada la fiabilidad se procede a aplicar 385 cuestionarios con 21 preguntas o variables, en las zonas vulnerables del Cantón Santo Domingo y de manera aleatoria se aplicaron 271, con similares preguntas en La Concordia.

Tabla 1. Estadística de Factibilidad

$\frac{\text { Alfa de Cronbach }^{\mathbf{a}} \quad \text { Número de elementos }}{21}$

Las principales técnicas que se emplearon fueron la observación, la documentación, la encuesta y la entrevista; mientras que los instrumentos dependieron de la técnica, para el caso de la observación, fue un registro diario, agenda, cuaderno de apuntes, filmadoras, cámaras fotográficas y otros dispositivos electrónicos. Para la encuesta, los instrumentos fueron un cuestionario, con preguntas cerrados, dicotómicas y politómicas, tipo escala de Likert.

La información primaria que se recolectó durante la investigación desarrollada en los cantones de Santo Domingo y La Concordia, mediante la aplicación del cuestionario de 21 preguntas contenidas en el instrumento de recolección de datos, y luego de la identificación y examinación, fueron procesados, tabuladas y analizadas mediante el paquete estadístico SPSS v 25, herramienta de importancia para el manejo de datos.

Fue necesario para el análisis de datos que se utilice el programa informático Excel, para la elaboración de tablas y figuras con la respectiva interpretación y análisis estadístico. Morán y Alvarado (2013). Sostienen que el análisis de "los datos recolectados mediante cuestionarios, entrevistas, escala de actitudes, observación, grupos de enfoques u otros medios deben analizarse para responder las preguntas 
de investigación y probar o descartar la hipótesis" (p. 76). Para aprobar o negar la hipótesis se procederá con el estadístico no paramétrico y el coeficiente de correlación de Spearman.

El instrumento de recogida de datos fue elaborado en base al principio "el instrumento mide lo que el investigador quiere que mida", de tal manera que este instrumento fue elaborado y pensado para que sea de utilidad para poder determinar de mejor manera el constructo o la construcción teórica del problema científico que se trata de resolver.

\section{RESULTADOS Y DISCUSIÓN}

La investigación que se ha puesto en consideración a la comunidad científica nacional e internacional, tiene un alto grado de innovación, pues si bien es ciento, en muchos países del mundo, se ha investigado el tema del presupuesto participativo, ha sido aplicado en la administración municipal con favorables resultados, y de igual manera en el Ecuador.

De un total de 21 preguntas que figuraron en el cuestionario de la encuesta aplicada, para efecto de este informe se han considerado que son cuatro las que mayormente aportan a la investigación, de tal manera, que se realizarán explicaciones breves de los datos estadísticos obtenidos, al tiempo que se realizó breve reseñas de los resultados obtenidos en cada pregunta.

Tabla 2. ¿Considera necesario que las autoridades municipales y la ciudadanía tenga conocimiento de lo planteado por la Constitución de la República, sobre el presupuesto participativo?

\begin{tabular}{llcccc}
\hline & FRECUENCIA & PORCENTAJE & $\begin{array}{c}\text { PORCENTAJE } \\
\text { VÁLIDO }\end{array}$ & $\begin{array}{c}\text { PORCENTAJE } \\
\text { ACUMULADO }\end{array}$ \\
\hline \multirow{2}{*}{ Muy importante } & 220 & 57,1 & 57,1 & 57,1 \\
Válido & 98 & 25,5 & 25,5 & 82,6 \\
& Importante & 33 & 8,6 & 8,6 & 91,2 \\
& Poco Importante & 12 & 3,1 & 3,1 & 94,3 \\
& Algo importante & 22 & 5,7 & 5,7 & 100,0 \\
& Sin importancia & $\mathbf{3 8 5}$ & $\mathbf{1 0 0 , 0}$ & $\mathbf{1 0 0 , 0}$ & \\
\hline
\end{tabular}

Fuente: Elaboración propia de los autores, procesamiento información (2020)

El $57,1 \%$ que equivale a 220 personas, respondieron que es muy importante que tengan conocimiento y que la apliquen en la gestión pública municipal, el 25,5\% de los consultados que es importante que tengan conocimiento pleno de lo que establece la carta constitucional, el 8,6\% que es poco importante y al mismo tiempo el 5,7\% considera esta situación sin importancia y el 3,1\%, asumen que es algo importante. 
Tabla 3. ¿Cree usted que las autoridades y la ciudadanía, deberían conocer lo planteado por el Código Orgánico de Organización Territorial, Autonomía y Descentralización COOTAD, sobre el presupuesto participativo?

\begin{tabular}{llcccc}
\hline & FRECUENCIA & PORCENTAJE & $\begin{array}{c}\text { PORCENTAJE } \\
\text { VÁLIDO }\end{array}$ & $\begin{array}{c}\text { PORCENTAJE } \\
\text { ACUMULADO }\end{array}$ \\
\hline \multirow{2}{*}{ Muy importante } & 195 & 50,6 & 50,6 & 50,6 \\
\multirow{2}{*}{ Válido } & 90 & 23,4 & 23,4 & 74,0 \\
& Importante & 48 & 12,5 & 12,5 & 86,5 \\
& Poco Importante & 34 & 8,8 & 8,8 & 95,3 \\
& Algo importante & 18 & 4,7 & 4,7 & 100,0 \\
\hline
\end{tabular}

Fuente: Elaboración propia de los autores, procesamiento información (2020)

Se les consulta a los encuestados si consideran que las autoridades deberían conocer el planteamiento que hace el COOTAD, sobre el presupuesto participativo, el 50,6\% considera que es de mucha importancia para poder aplicarlo en su gestión, de igual manera el $23,4 \%$ considera que es importante su conocimiento, mientras que el $4,7 \%$ considera sin importancia.

Tabla 4. ¿Considera necesario que la ciudadanía y las autoridades municipales tengan conocimientos de la Ley Orgánica de Participación Ciudadana, sobre el presupuesto participativo?

\begin{tabular}{clcccc}
\hline & FRECUENCIA & PORCENTAJE & $\begin{array}{c}\text { PORCENTAJE } \\
\text { VÁLIDO }\end{array}$ & $\begin{array}{c}\text { PORCENTAJE } \\
\text { ACUMULADO }\end{array}$ \\
\hline \multirow{2}{*}{ Muy importante } & 180 & 46,8 & 46,8 & 46,8 \\
Válido & 129 & 33,5 & 33,5 & 80,3 \\
& Importante & 37 & 9,6 & 9,6 & 89,9 \\
& Poco Importante & 28 & 7,3 & 7,3 & 97,1 \\
& Algo importante & 11 & 2,9 & 2,9 & 100,0 \\
& Sin importancia & $\mathbf{3 8 5}$ & $\mathbf{1 0 0 , 0}$ & $\mathbf{1 0 0 , 0}$ & \\
\hline
\end{tabular}

Fuente: Elaboración propia de los autores, procesamiento información (2020)

La encuesta implementada buscaba conocer la opinión de las personas sobre su opinión de que las autoridades municipales tengan conocimiento de la Ley Orgánica de Participación Ciudadana, el 46,8\% considera que es de mucha importancia y de igual manera el 33,5\% considera importante, mientras que el $2,9 \%$ considera sin importancia, se vincula con la variable dependiente.

Tabla 5. ¿Será necesario, según su opinión, que las autoridades municipales cumplan con lo que establece la Ley Orgánica de Planificación y Finanzas Públicas, sobre el presupuesto participativo?

\begin{tabular}{llcccc}
\hline & FRECUENCIA & PORCENTAJE & $\begin{array}{c}\text { PORCENTAJE } \\
\text { VÁLIDO }\end{array}$ & $\begin{array}{c}\text { PORCENTAJE } \\
\text { ACUMULADO }\end{array}$ \\
\hline \multirow{6}{*}{ Válido } & Muy importante & 155 & 40,3 & 40,3 & 40,3 \\
& Importante & 149 & 38,7 & 38,7 & 79,0 \\
& Poco Importante & 33 & 8,6 & 8,6 & 87,5 \\
& Algo importante & 29 & 7,5 & 7,5 & 95,1 \\
& Sin importancia & 19 & 4,9 & 4,9 & 100,0 \\
& Total & $\mathbf{3 8 5}$ & $\mathbf{1 0 0 , 0}$ & $\mathbf{1 0 0 , 0}$ & \\
\hline
\end{tabular}


Fuente: Elaboración propia de los autores, procesamiento información (2020)

El 46,3\% que equivale a 155 personas consideran que es muy importante que el gobierno local tenga conocimiento pleno de estas disposiciones legales para cumplir con su responsabilidad, el 38,7\% considera que es importante que las autoridades y la ciudadanía tengan conocimiento de las leyes, el 8,6\% considera que es poco importante, mientras que el 7,5\%, aseguró que es algo importante, un reducido número de personas de 19, que equivale al 4,9\% consideró sin importancia el conocimiento de la ley.

\subsection{RESULTADOS DE LA EXPERIMENTACIÓN SOCIAL}

La experimentación de campo o experimentación social es parte de la investigación explicativa, que según Hernández Sampieri et al (1991) "la mayoría de las investigaciones explicativas desarrolladas son experimentales" ya que mediante este ejercicio investigativo se aporta con evidencia cierta, que permiten entender de mejor manera el fenómeno en estudio. Las ciencias sociales, así como las ciencias económicas, las ciencias políticas, así como las ciencias de la Psicología y la de la Sociología, son las que mayormente han aportado en este campo de la investigación.

Con la intención de encontrar mejores resultados, se realizó una experimentación de campo, entendiendo que dentro de la investigación social, esta nueva forma ha tomado mucha fuerza y se lo ha denominado estudio experimental o el diseño experimental y también conocido como la experimentación de campo, esta es una forma de estudio investigativo que ha adquirido mucha utilidad ya que permite responder de manera asertiva a la problemática planteada, lo cual enriquece de forma sustantiva los resultados obtenido del fenómeno estudiado.

La experimentación de campo en las ciencias sociales, busca crear un escenario acorde para la interacción de los involucrados, "La tarea es crear una situación artificial similar en ciertas características a la de las situaciones reales de la vida social, pero que cumpla con los criterios, que pueda haber manipulación de variables en el surgimiento de un fenómeno social" (González, Cabrera, Mendoza y Arzate, 2020, p. 112).

\subsubsection{Laboratorio social uno}

Tabla 6. Experimento social laboratorio número uno

\begin{tabular}{lcc}
\hline \multicolumn{1}{c}{ PARTICIPANTES } & \# DE & PORCENTAJE \\
\hline Hombres & PARTICIPANTES & $46 \%$ \\
Mujeres & 23 & $54 \%$ \\
Total & $\mathbf{5 0}$ & $\mathbf{1 0 0 \%}$ \\
\hline \multicolumn{2}{l}{ Fuente: Elaboración propia de los autores, procesamiento información (2020) }
\end{tabular}


Laboratorio social número uno, se relacionó a grupos humanos diversos, 23 hombres y 27 mujeres de diferentes edades, en un total de 50 personas, todos habitantes de sectores vulnerables de los cantones de Santo Domingo y La Concordia del área urbano marginal y que se ha determinado que carecen de los servicios básicos y de obras de desarrollo elementales, quienes participaron tenían poco o nada de conocimientos acerca del presupuesto participativo y de la participación ciudadana, y ningún momento han sido invitados a participar en asambleas ciudadanas por las autoridades locales.

\subsubsection{Laboratorio social dos}

Tabla 7. Experimento social laboratorio número dos

\begin{tabular}{lcc}
\hline \multicolumn{1}{c}{ PARTICIPANTES } & \# DE & \multirow{2}{*}{ PORCENTAJE } \\
\hline Hombres & PARTICIPANTES & $59 \%$ \\
Mujeres & 26 & $41 \%$ \\
Total & 18 & $\mathbf{1 0 0 \%}$ \\
\hline \multicolumn{2}{c}{ Fuente: Elaboración propia de los autores, procesamiento información (2020) }
\end{tabular}

Para el desarrollo del laboratorio social número dos, fue necesaria la participación de grupo humano perteneciente a otros sectores vulnerables que se encuentran asentados en el área urbana marginal, se tuvo la participación de 44 de las 60 personas que fueron invitadas con la debida anticipación y que habían comprometido su asistencia. Entre los asistentes al evento en donde se pondría en práctica una experiencia de participación ciudadana y de elaboración del presupuesto participativo, se encontraban personas de ambos sexos y de diferentes edades, 26 hombres y 18 mujeres.

Tabla 8. Perfiles de participantes en laboratorios sociales

\begin{tabular}{lcc}
\hline \multicolumn{1}{c}{ FORMACIÓN } & PCARTICIPANTES & PORCENTAJE \\
\hline Formación básica & 15 & $26 \%$ \\
Estudios secundarios & 29 & $50 \%$ \\
Bachillerato y técnico & 10 & $17 \%$ \\
Universitario y cuarto nivel & 4 & $7 \%$ \\
$\quad$ Total participantes & $\mathbf{5 8}$ & $\mathbf{1 0 0 \%}$ \\
\hline \multicolumn{2}{c}{ Fuente: Elaboración propia de los autores, procesamiento información (2020) }
\end{tabular}

Fuente: Elaboración propia de los autores, procesamiento información (2020)

En cada sesión se contó con personas de diferentes lugares de la zona urbano marginal de los cantones estudiados, en los dos grupos los perfiles de participantes eran similares, algunos tenían experiencias en eventos participativos. Se destaca que en cada sesión se contó con un pequeño grupo de personas cómplices que conocían del experimento social que se estaba llevando a cabo y otro grupo que 
simulaba ser miembros del Gobierno Local, y al mismo tiempo, la mayoría de los participantes desconocían de lo que se trataba.

\subsubsection{Resultados y Hallazgos de laboratorio social}

La participación ciudadana de diversos actores sociales que tuvo lugar en la experimentación de campo, en donde pobladores y vecinos se encontraron para entablar conversaciones y compartir experiencias acerca de las necesidades insatisfechas y prioritarias comunes, que necesitan para su solución la intervención urgente de parte del Gobierno Municipal local.

El laboratorio social uno evidenció una escasa participación de los miembros participantes y esto es entendible, por cuanto ellos desconocían cuales eran los derechos y obligaciones que las leyes vigentes sobre el presupuesto participativo les otorga. En el laboratorio dos existió mayor discusión y planteamientos de necesidades por parte de los miembros de la comunidad presente, reclamaron mayor participación en las decisiones de políticas públicas que adopte el Gobierno Municipal, de los resultados se puede colegir, que entre más conocimiento tiene la ciudadanía mayor es la posibilidad de acceder a las obras prioritarias y de servicios básicos.

Frente a esta acción se determina que el conocimiento en todo momento y en cada lugar es una expresión de poder y si la ciudadanía no tiene conocimientos y si este conocimiento es negado por la autoridad municipal, las posibilidades de desarrollo que garanticen mejores condiciones de vida para los sectores marginales de Santo Domingo y La Concordia se ve muy lejana.

\subsection{EVALUACIÓN DE LA HIPÓTESIS}

Según Hernández, Fernández, y Baptista (2014), “Las hipótesis se someten a prueba o escrutinio empírico para determinar si son apoyadas o refutadas, de acuerdo con lo que el investigador observa" por tanto la investigación empírica realizada ha sido sometida a evaluación mediante el instrumento estadístico del coeficiente de correlación de Spearman (p. 107).

\subsubsection{Prueba de Hipótesis no paramétrica}

H1: Las deficiencias en la gestión del presupuesto participativo son provocadas por la debilidad de un modelo de gestión de presupuesto participativo en los gobiernos autónomos descentralizados municipales.

H0: Las deficiencias en la gestión del presupuesto participativo, no son provocadas por la debilidad de un modelo de gestión de presupuesto participativo en los gobiernos autónomos descentralizados municipales. 
Se utilizó SPSS para determinar la relación existente entre las variables de estudio que da como resultado un valor Rho de Spearman de 0,126, y es a la vez un valor positivo, y al mismo tiempo se determina que el $\mathbf{p}$ valor es de 0,013 también positivo, por tanto, se establece que la correlación existente es significativa en el nivel del 0,05.

Tabla 9. Prueba de hipótesis coeficiente correlación de Spearman

\begin{tabular}{|c|c|c|c|c|}
\hline \multicolumn{5}{|c|}{ CORRELACIONES } \\
\hline & & & $\begin{array}{c}\text { Las deficiencias en la } \\
\text { gestión del } \\
\text { presupuesto } \\
\text { participativo son } \\
\text { provocadas por la } \\
\text { debilidad de un } \\
\text { modelo de gestión? }\end{array}$ & $\begin{array}{l}\text { ¿ Las deficiencias en la } \\
\text { gestión del presupuesto } \\
\text { participativo, no son } \\
\text { provocadas por la } \\
\text { debilidad de un modelo de } \\
\text { gestión? }\end{array}$ \\
\hline \multirow{6}{*}{$\begin{array}{l}\text { Rho de } \\
\text { Spearrman }\end{array}$} & $\begin{array}{l}\text { ¿ Las deficiencias } \\
\text { en la gestión del }\end{array}$ & $\begin{array}{l}\text { Coeficiente de } \\
\text { correlación }\end{array}$ & 1,000 &, $126^{*}$ \\
\hline & $\begin{array}{l}\text { presupuesto } \\
\text { participativo son } \\
\text { provocadas por la }\end{array}$ & Sig. (bilateral) & . & 013 \\
\hline & $\begin{array}{l}\text { debilidad de un } \\
\text { modelo de gestión? }\end{array}$ & $\mathrm{N}$ & 385 & 385 \\
\hline & $\begin{array}{l}\text { ¿Las deficiencias en } \\
\text { la gestión del }\end{array}$ & $\begin{array}{l}\text { Coeficiente de } \\
\text { correlación }\end{array}$ &, $126^{*}$ & 1,000 \\
\hline & $\begin{array}{l}\text { presupuesto } \\
\text { participativo, no } \\
\text { son provocadas por }\end{array}$ & Sig. (bilateral) & ,013 & . \\
\hline & $\begin{array}{l}\text { la debilidad de un } \\
\text { modelo de gestión? }\end{array}$ & $\mathrm{N}$ & 385 & 385 \\
\hline
\end{tabular}

Fuente: Elaboración propia de los autores, procesamiento información (2020)

Con los datos obtenidos se procede a realizar la comparación existente entre el p valor con el alfa, que como se ha señalado es equivalente al 5\%, que es el margen de error que está dispuesto a aceptar el investigador, por tanto, se tendría que: $\mathrm{p}$ valor $=0,013<\alpha=0,05$ y el establecimiento de una correlación directa entre variables. Por tanto, en la comparación se establece que el p valor es menor al valor de alfa, con lo cual se procede a tomar la decisión de rechazar la hipótesis nula (Ho).

Se concluye mediante la correlación Rho Spearman que existe una correlación entre las variables del conocimiento que tienen los encuestados sobre el presupuesto participativo, al mismo tiempo se observa que el valor probabilístico o el valor de significancia está por debajo de 0,05 es decir que existe significancia estadística suficiente para proceder a rechazar la hipótesis nula y tomar la hipótesis alternativa o del investigador, implica que existe una buena correlación entre las variables y una alta covarianza en el tema de investigación propuesto. 


\section{CONCLUSIONES}

Los gobiernos autónomos descentralizados cantonales de Santo Domingo y de La Concordia, tienen un gran reto en el desarrollo de la administración pública por mandato de la ley, la de elaborar sus presupuestos anuales de manera participativa; si bien, hacen un llamado a los miembros de los concejos barriales, para que participen en las asambleas, han creado departamentos de participación ciudadana, aun así, coexiste una debilidad en la gestión al no haber utilizado esta herramienta para resolver los problemas prioritarios de la población de los sectores vulnerables, sino más bien lo han hecho de manera discrecional, para "cumplir con la ley".

Los resultados obtenidos de la encuesta, entrevistas y la experimentación social deja en evidencia que a pesar de que las autoridades municipales sostengan que el desarrollo de la administración pública, se está elaborando de forma participativa su presupuesto anual, la ciudadanía y los líderes sociales consideran lo contrario, por tanto, existe una debilidad en la gestión, pues del total de los encuestados la gran mayoría desconoce de qué se trata el presupuesto participativo. Se debe de establecer la propuesta a través de un enfoque de desarrollo humano como horizonte posible de formación de capacidades y competencias, según los criterios expuestos por Villa (2016).

Los recursos económicos que ingresan a los gobiernos municipales, vía impuestos, tasas y contribuciones y las asignaciones estatales no son distribuidos de manera equitativas, participativas y solidaria, sobre todo en los sectores vulnerables y es producto de las deficiencias que tiene el Gobierno municipal al momento de elaborar el presupuesto anual.

La falta de obras básicas en los sectores vulnerables, está relacionada de manera directa con la elaboración del presupuesto de manera participativa, por tanto, la deficiencia estaría en que los gobiernos autónomos descentralizados municipales no elaboran el presupuesto anual con la ciudadanía y por ello no se atienden las necesidades prioritarias.

Existe un marco legal amplio y propicio para que los gobiernos locales elaboren su presupuesto de forma participativa, pero en los hechos no se lo hace y, de acuerdo a los datos obtenidos en la encuesta, es fundamentalmente por la deficiencia o carencia de un modelo de gestión simplificado que permita de forma didáctica construir entre la ciudadanía y la entidad municipal el presupuesto participativo, con lo cual, se impediría que cada gobierno local lo haga según su criterio y con ello, se desaprovecha este efectivo instrumento administrativo y financiero.

Las autoridades municipales, de acuerdo a los hallazgos en la investigación aprovechando el desconocimiento de la ciudadanía, no respetan y no ponen en práctica lo establecido por la Constitución de la República, el COOTAD, Ley Orgánica de Participación Ciudadana, Código Orgánico de 
planificación y finanzas públicas, en lo relacionado con la elaboración del presupuesto de manera participativa.

Visitando en territorio en donde se encuentra la población vulnerable de Santo Domingo y la Concordia, se observa que existe una ausencia o deficiencia de los servicios y necesidades prioritarias como los servicios públicos del agua potable, alcantarillado, aguas residuales, manejo de basura o de desechos sólidos, saneamiento ambiental, transporte público, infraestructura básica y equipamiento de salud y espacios físicos para el desarrollo cultural, social y deportivo, propias de las competencias de los gobiernos municipales; con lo que, se manifiesta una mala distribución de los recursos económicos propios y de los que ingresan por asignaciones del Gobierno Nacional.

La acción deficiente de la administración pública en los gobiernos municipales en el desarrollo del presupuesto participativo, de acuerdo a la investigación realizada, no permite que las personas que viven en los sectores considerados vulnerables económicamente y la sociedad en su conjunto, participen al tenor de la ley, con sus líderes locales, en la elaboración, ejecución y evaluación del presupuesto anual. 


\section{BIBLIOGRAFÍA}

(2015). Agenda 2030. Objetivos de Desarrollo Sostenible ONU.

(2008). Constitución de la República del Ecuador, Asamblea Nacional Constituyente de MontecristiEcuador.

(2010). INEC, Instituto Nacional de Estadísticas y Censo.

Ayala, L. (2015). Gestión Social Comunitaria: Rol del Presupuesto Participativo, caso: GAD de la Parroquia Llano Chico. (Tesis Magister) PUCE.

Buele Nugra, I., Vidueira, P. (2018). Presupuesto Participativo: una revisión de la investigación científica y sus implicaciones democráticas del 2000 al 2016. Universitas, 28, pp. 159-176. https://doi.org/10.17163/uni.n28.2018.08

Carmona, R., Martínez, C. (2014). Luces y sombra del presupuesto participativo en la región metropolitana de Buenos Aires. Región y Sociedad, 26 (61), 267-308. http://www.scielo.org.mx/scielo.php?script=sci_abstract\&pid=S1870-

$39252014000400008 \& \operatorname{lng}=$ es\&nrm=iso

Díaz, Pablo Ignacio. (2011). El presupuesto participativo en la gestión local: El caso del municipio de Maipú en la Provincia de Mendoza (Argentina) (Tesina de grado). Mendoza, Universidad Nacional de Cuyo. Facultad de Ciencias Políticas y URL del informe: https://bdigital.uncu.edu.ar/5970 Fecha de consulta del artículo: 10/12/20.

Garrido, F.; Montecinos, E. (2018). Presupuesto Participativo en Chile y República Dominicana; ¿Es determinante una ley para el fortalecimiento de la democracia participativa? Revista Uruguay de Ciencia Política, 27 (2), pp. 99-120. https://hdl.handle.net/20.500.12008/19505

Hernández, R.; Mendoza, P. (2018). Metodología de la Investigación. Las rutas Cuantitativa, Cualitativa y Mixta. México: Mc Graw Hill.

Hidalgo, P. (2017). Democracia Participativa, Proximidad y Capacidad Organizativa: el caso de las Asambleas de Presupuesto Participativo del Distrito Metropolitano de Quito 2010-2013. (Tesis Maestría). FLACSO-ECUADOR.

León, A; Hincapié, L. y Villa, G. (2016). Potencial Social y Político de la Planeación Local y el Presupuesto Participativo en Medellín (Colombia) para Fortalecer la Democracia latinoamericana. EURE (Santiago), 42 (125), pp. 205-224. http://dx.doi.org/10.4067/S0250-71612016000100009.

Montecinos, E. (2012). Democracia y Presupuesto Participativo en América Latina. La Mutación del Presupuesto Participativo fuera de Brasil. Revista del CLAD Reforma y Democracia, 53, 61-96. Centro Lat. de Administración para el Desarrollo Caracas, Venezuela. https://www.redalyc.org/pdf/3575/357533685003.pdf

Morán, G.; Alvarado, D. (2013). Métodos de Investigación. México: Pearson.

Nebot, C. (2018). El Presupuesto Participativo de Madrid: Luces y Sombras. Revista de Gestão e Secretariado, 9 (1), 265-292. DOI: https://doi.org/10.7769/gesec.v9i1.687 
Noriega, A.; Aburto, F. y Montecinos, E. (2016). Presupuesto Participativo en Chile y su Contribución a la Inclusión Social. Iconos Revista de Ciencias Sociales, 56, pp. 203218. https://www.redalyc.org/articulo.oa?id=50947321011

Pilay, F. (2015). Presupuesto Participativo para una Efectiva Participación Ciudadana. Revista, ECA Sinergia, 6 (1), 69-76. Universidad Técnica de Manabí. https://dialnet.unirioja.es/servlet/articulo?codigo=6197617

Pilay, F., Ugando, M. (2020). Presupuesto participativo en gobiernos autónomos descentralizados municipales de la provincia Santo Domingo de los Tsáchilas - Ecuador. Revista, ECA Sinergia, 11 (3), 137-151. Universidad Técnica de Manabí. https://revistas.utm.edu.ec/index.php/ECASinergia/article/view/2729/2859 DOI: https://doi.org/10.33936/eca_sinergia.v11i3.2729

Ugando, M., Jurado, Z., \& Cedeño, A. (2021). Implementación de la planificación estratégica para empresas del sector automotriz en la ciudad de Santo Domingo de los Tsáchilas, Ecuador: Caso Importadora de Autorepuestos Mendoza. South Florida Journal of Development, Miami, v.2, (4), jul./sep. p. 5017-5033. https://doi.org/10.46932/sfjdv2n4-006

Villa, G.L. (2016). El desarrollo humano integral. Horizonte de formación por capacidades y competencias. La gestión del proyecto institucional universitario. Espiral, Revista de Docencia e Investigación, 6(1), 9 - 58. DOI: https://doi.org/10.15332/erdi.v6i1.1584

Welp, Y. (2016). La participación ciudadana como compromiso democrático. Revista Mexicana de Derecho Electoral, 10, 97-121. DOI: http://dx.doi.org/10.22201/iij.24487910e.2016.10.11122 